CC Springer-Verlag London Ltd Virtual Reality (1999) 4:169-183

\title{
Virtual Reality in Vestibular Assessment and Rehabilitation
}

\author{
S. Di Girolamo', W. Di Nardo', P. Picciotti', G. Paludetti', \\ F. Ottaviani ${ }^{1}$, O. Chiavola ${ }^{2}$
}

'Institute of Otorhinolaryngology, Catholic University of the Sacred Heart; ${ }^{2}$ Department of Mechanical and Industrial Engineering, Romatre University, Rome, Italy

\begin{abstract}
Previous experiences on vestibular compensation showed that multisensorial stimulations affect postural unbaiance recovery. Virtual Environment (VE) exposure seems very useful in vestibular rehabilitation, since the experience gained during VE exposure is transferable to the real world. The rearrangement of the hierarchy of the postural cues was evaluated in 105 patients affected by visual, labyrinthic and somatosensory pathology in nomal conditions and during sensoria deprivation. They were divided into five groups according to pathology and compared to 50 normal controls. Our data show that VE exposure is a reliable method to identify the deficjent subsystem and the level of substitution. Moreover. Virtual Reality (VR) would accelerate the compensation of an acute loss of labyrinthine function, related to adaptive modifications of the vestibulo-ocular and vestibulo-spinal reflexes, overstimulating the residual labyrinthine function. The residual labyrinthine function is poor in chronic bilateral vestibular deficit and VE exposure should provide sensory substitution or sensory motor reorganisation, thereby modulating the external spatial reference and promoting the reorganisation of the multiple sensory input. The potential for VE exposure perspectives seems very promising when dealing with the vestibular system where there is a continuous rearrangement of different sensorial informations as a result of environmental and age-related changes.
\end{abstract}

Keywords: Postural control; Virual Reality; Vestibular assessment; Vestibular rehabilitation

\section{Introduction}

The aim of this paper was to analyse the rela. tionships between the vestibular system and the virtual world in order to sudy the possible applica tion of virtual reality (VR) in the assessment and rehabilitation of vestibular system dysfunctions. The vestibular system controls the continuously changing relationships of our body with the environment and modulates the activity of the different subsystems in order to ensure optimal postural control and a stable gaze. Any pathology affecting this system can be very disabling, infact a hypofunction of the labyrinthine, visual and/or somatosensory afferences causes an unbalance of the two vestibular subsystems. The clinical manifestations are characterised by postural and visual disturbances, similar to those often reported during and after VR exposure $[1,2,3]$. Virtual environment (VE) related side effects are characterised by a condition of sensory rearrangement in which the motion signals transmitted by the eyes, the vestibular system, and the proprioreceptors are in conflict among themselves or with previous experiences (neural store). This sensory conflict is probably related to an incongruity among different sense perception modalities, and in particular between what is 'expected' versus what is the individual's sensory experience in the VE enviromment [4]. In normal subjects these effects result from the 
mismatches between visual perception (apparent movement in the virtual environment) and the somatosensorial and labyrinthine informations experienced in the physical reality. Therefore, these symptoms mainly represent a cue conflict between the vestibular and visual systems. Similar experiences have been widely observed in aircraft simulators [2].

Virtual environment-related side effects are commonly described as cybersickness and aftereffects $[5,6]$

Cybersickness occurs during VE exposure [7] and is characterised by nausea, vomiting, eyestrain, disorientation and vertigo [8]. The signals coming from the proprioceptive system and the external signals of a virtual environment may atter body perception and cause discomfort [9]. These disturbances may be due to a difficult adaptation to the sensory/motor requirements of the $V E[10,11]$. Normal sensorimotor loops are altered by the presence of distortion time delays and noise.

Aftereffects appear after VE exposure and include disturbed locomotion, changes in postural control, perceptual-motor disturbances, pastpointing, flashbacks, drowsiness, fatigue and generally lowered arousal. The occurrence of aftereffects may reflect the lack in the readaptation to the nonVE environment. They might depend on a shift in sensory-motor response recalibration.

The occurrence of side effects in virtual environments varies across studies [12,13], depending upon:

1. the cype of VE programme that is used:

2. the technical drivers:

3. the length of the exposure time;

4. the individual's prior VE experience;

5. active vs. passive movement;

6. gender;

7. the method of measurements used to assess occurrence

In one study, $61 \%$ of 146 healthy subjects reported 'symptoms of malaise' at some point during a 20 minute immersion and 10 -minute postimmersion period [5]. In a recent study, the occurrence of side effects was 95\% [3]. For this reason Rizzo and Buckwalter [14] suggested that particular concern might be directed towards neurologically impaired populations, some of whom may manifest longterm equilibrium, balance and orientation difficulties.

Paradoxically, VR exposure can be used as a new therapeutic approach to overcome some of the difficulties encountered in the treatment of vestibular disorders.
Our sense of physical reality is, in fact, a construction derived from the symbolic, geometric and dynamic information directly presented to our senses and elaborated by the vestibular system. The output channels of a VR application can stimulate the peripheral receptors of our senses: vision, hearing, touch and force perception. Therefore, VR can be used to evaluate and stimulate the systems playing a role in a human's perception of (sensory) motion in order to induce and accelerate the vestibular rearrangements leading to reduction of vestibular symptoms (vertigo and dizziness) [15].

\section{Vestibular System}

The vestibular system provides the central nervous system with information concerning the reflex control of eye movements, the reflex control of upright posture, the body position and acceleration. It can be regarded as a circular, time-variant, causal and complex cybernetic system with the following characteristics:

- Totality. Every component is interrelated with the others, and any change of one component induces changes in all system elements.

- Equifinality. In such a system it is more important how the subsystems communicate among themselves than their single state. Different interconnections and different states of the single subsystems may achieve the same functional result. These rules strongly imfluence the neurophysiologic course of vestibular compensation $[16,17]$.

- Feedback. The system must be always informed on the state of its outputs and subsystems in order to provide their regulation.

- Calioration. The system is stable towards its inputs if they stay within predetermined limits. This law represents the tolerance of the system to environmental and state changes of the subsystems.

- Redundancy. The vestibular system is polysensorial and many receptors send signals simultaneously concerning the same situation. The system is able to select in any moment the most useful sensorial information and motor outputs

- Preference. Any individual is able to develop a preferential sensorial-motor strategy in relation to age, possible sensorial deficits and previous sensorial experiences. 
Table 1. Vestibular system organisation.

\begin{tabular}{lll}
\hline Input & Output & Effect \\
\hline Labyrinth & Cortex & Spatial orientation \\
Semicircular canals & & Sensation of motion \\
Maculae & & Voluntary movements \\
Vision & VOR. & Gaze control \\
Proprioceptors & VSR & Postural control: neck, body limb tonus and movements \\
\hline
\end{tabular}

The human vestibular system is made up of three components: a peripheral sensory apparatus, a central processor, and a mechanism for motor output (Table 1). The peripheral apparatus consists of a set of motion sensors that send information to the central nervous system, specifically the vestibular nuclear complex and the cerebellum. Information concerning head angular velocity, linear acceleration and orientation of the head and body with respect to the gravitational axis are all part of this input. The central nervous system processes these signals and combines them with other sensory information to estimate head and body orientation. The output of the central vestibular system goes to the ocular muscles and spinal cord to serve two important reflexes: the vestibular-ocular reflex (VOR) and the vestibular-spinal reflex (VSR)

The VOR generates eye movements that enable clear vision while the head is in motion. The
VSR generates compensatory body movements to maintain head and postural stability, thereby preventing falls. Unlike the VOR, there is no single VSR but rather an assemblage of several reflexes that are dependent on the sensory and motor context. The performances of the VOR and VSR are monitored by the centra! nervous system and readjusted as needed by a rather complex adaptive processing mechanism.

\section{Labyrinthine Afferents}

The peripheral vestibular system includes the bony labyrinth, consisting of three semicircular canals and a central chamber, the vestibule containing the otolith organ (Fig. 1). They are biological sensors that convert displacement due to head motion into neural firing. Semicircular canals provide sensory

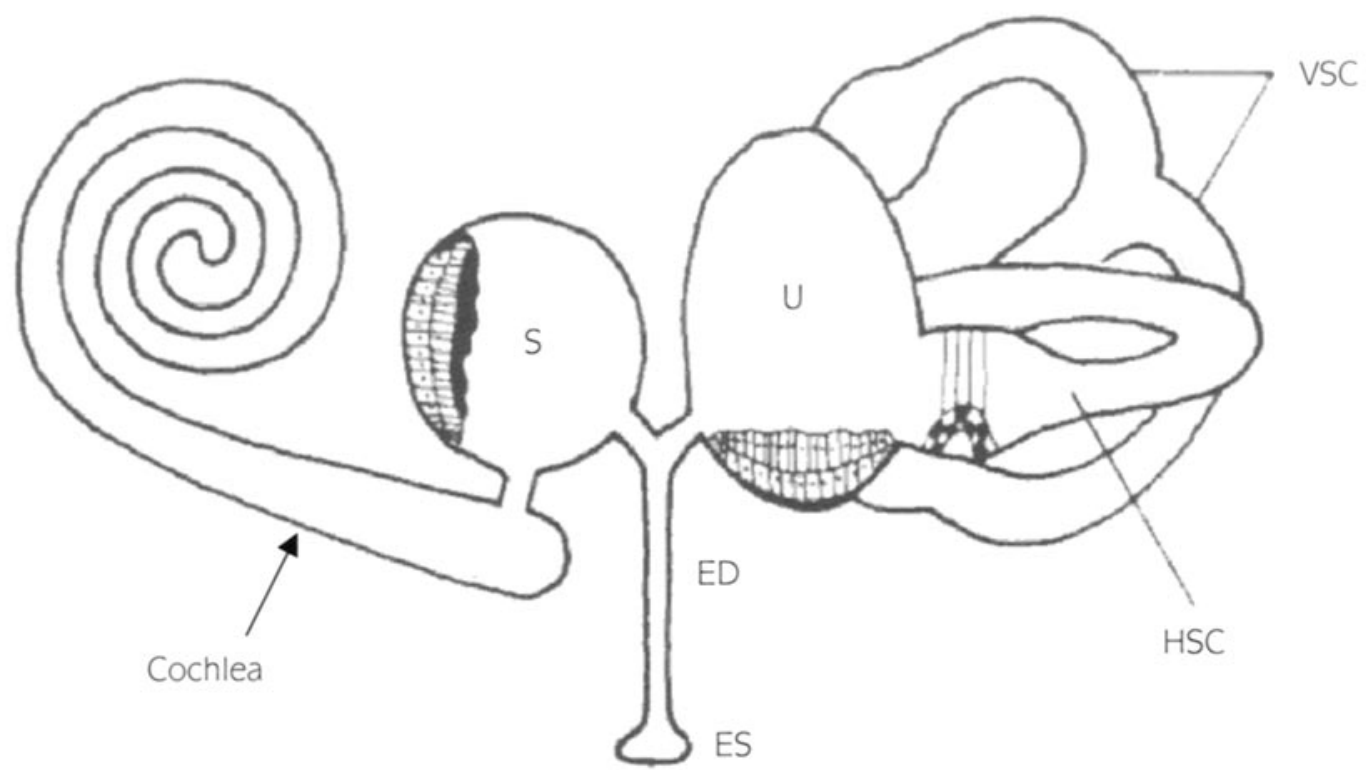

Fig. 1. Structure of labyrinthine receptors: U: Utricle and utricular macula; S: Sacculus and saccular macula; HSC: Horizontal Sernicircular Canal; VSC: Vertical Semicirculars Canals; ED: Endolymphatic duct; ES: Endolymphatic sac. 


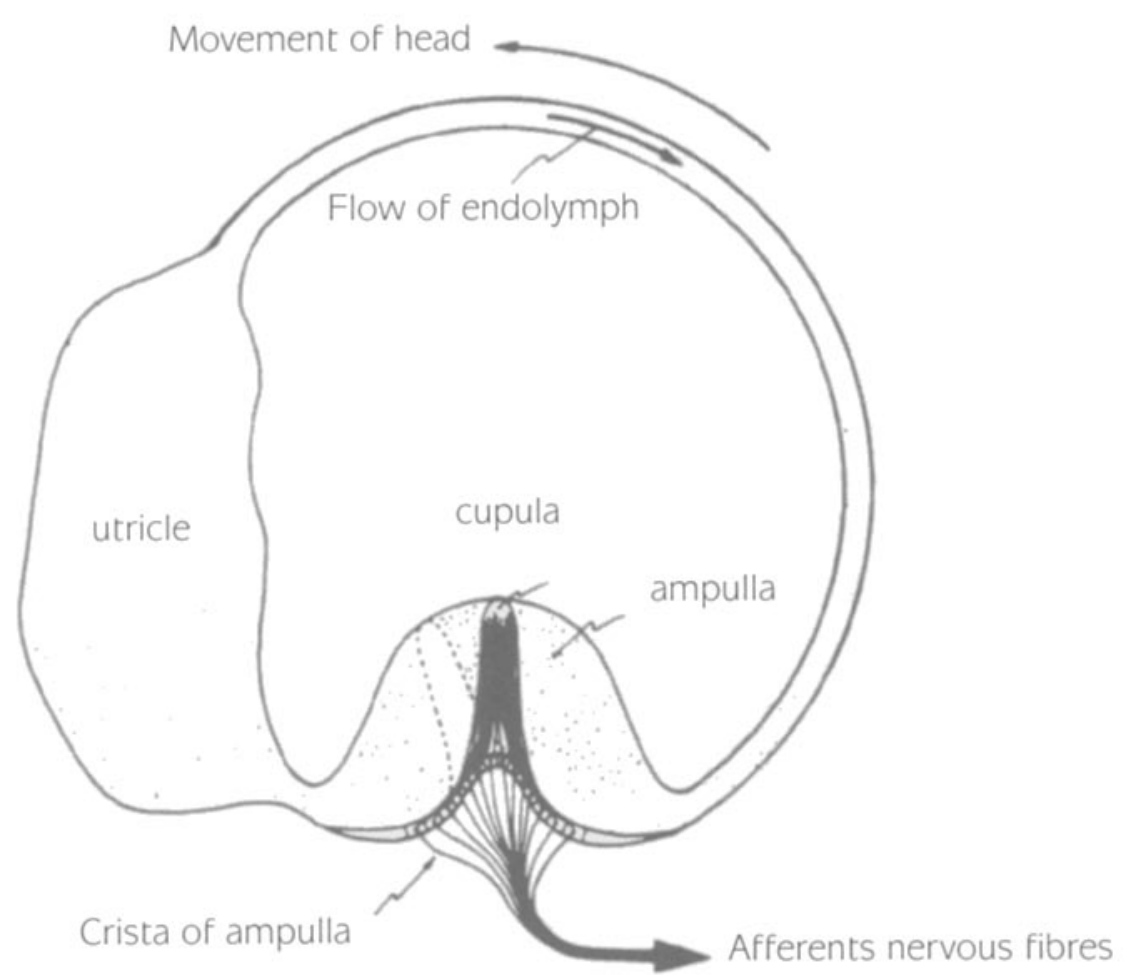

Fig. 2. Correlation between direction of head movement (big arrow). flow of endolymph (small arrow) and bending of cupula.

inputs about head angular velocity, which enable the VOR to generate an eye movement matching the velocity of the head movement. The result is that the eye remains stable in the space during head motion, enabling clear vision. The semicircular ducts provide information to the nervous system about angular acceleration and direction of head movement. As inertia is overcome, the duct fluids begin to move at the same rate as the head, and the hair cells slowly return to their resting position. For this reason, hair cells are stimulated only during changes in the rate of motion, i.e. during acceleration of the head (Fig. 2). The semicircular canals are arranged like closed circular tubes filled with a fluid, the endolymph. As the head rotates, this fluid moves relatively to the head. because of inertial forces. At the same time, the fluid movement is opposed by the viscous tube resistance and the cupola, which acts as a swing-door across the canal. The cupola itself provides slight inertial force as well as elastic and viscous resistance.

These conditions are similar to those of the 'torsion-pendulum' model of classical physics. Therefore, the movement of the cupola-endolymph unit relative to the head, can be expressed by a second order differential equation:
$\Theta \ddot{A}(t)+\Pi \dot{A}(t)+\Delta A(t)=\Theta I(t)$

where $\ddot{A}(t), \dot{A}(t)$ and $A(t)$ are cupola-endolymph angular acceleration, velocity and displacement, respectively; $\Theta$ is the cupola-endolymph unit moment of inertia; $\Pi$ is the cupola-endolymph viscous damping; $\Delta$ is the cupola-endolymph unit rotational stiffness and if(t) is the head angular acceleration. Values for $\Theta, \Pi$ and $\Delta$ can be estimated from the physical dimensions of the semicircular ducts and from the physical properties of the endolymph and cupola.

If we consider head movement in the frequency domain, the transfer function that relates cupola deflection to head angular acceleration can be obtained from the second order differential equation. This function contains two time constants that are different for different species. From the curves relating the cupola-endolymph system displacement to head acceleration, head velocity, and head position, it can be pointed out that for frequencies below $0.02 \mathrm{~Hz}$ the canals act as angular accelerometers, between 0.02 and $32 \mathrm{~Hz}$ as velocity meters, and above $32 \mathrm{~Hz}$ like position meters. Most normal head movements are in the range of 0.02-32 $\mathrm{Hz}$ and, therefore, the semicircular canals inform 


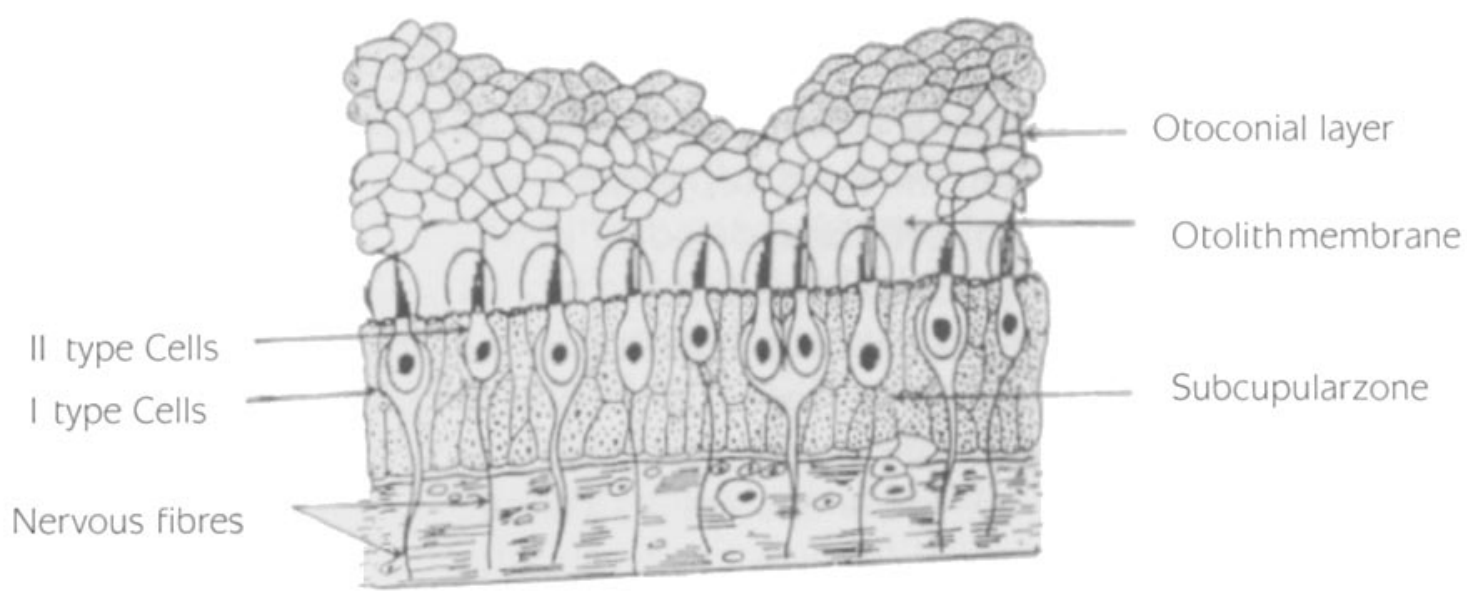

Fig. 3. Schematic rapresentation of utricular and saccular maculae.

the central nervous system behaving as head velocity meters.

The otoliths (Fig. 3) are made up by calcium carbonate crystals (otoconia, Fig. 4) and induce the displacement of the underlying neuroepithelium in response to linear acceleration [18]. They respond both to linear head motion and to static tit with respect to the gravitational axis. From the functional point of view all otolith receptors are best described as differential density accelerometers. The acequate stimulus for the macular hair cells is the tangential shearing force, which displaces the ciliary tufts and induces positive or negative shifts in receptor potentials. During linear acceleration, tangential to the otolith plane, otoconia (Fig. 3) are dislocated in opposite direction in respect to the head. This induces elastic deformation of the gelatinous layer resulting bending of the cilia. During head rotation

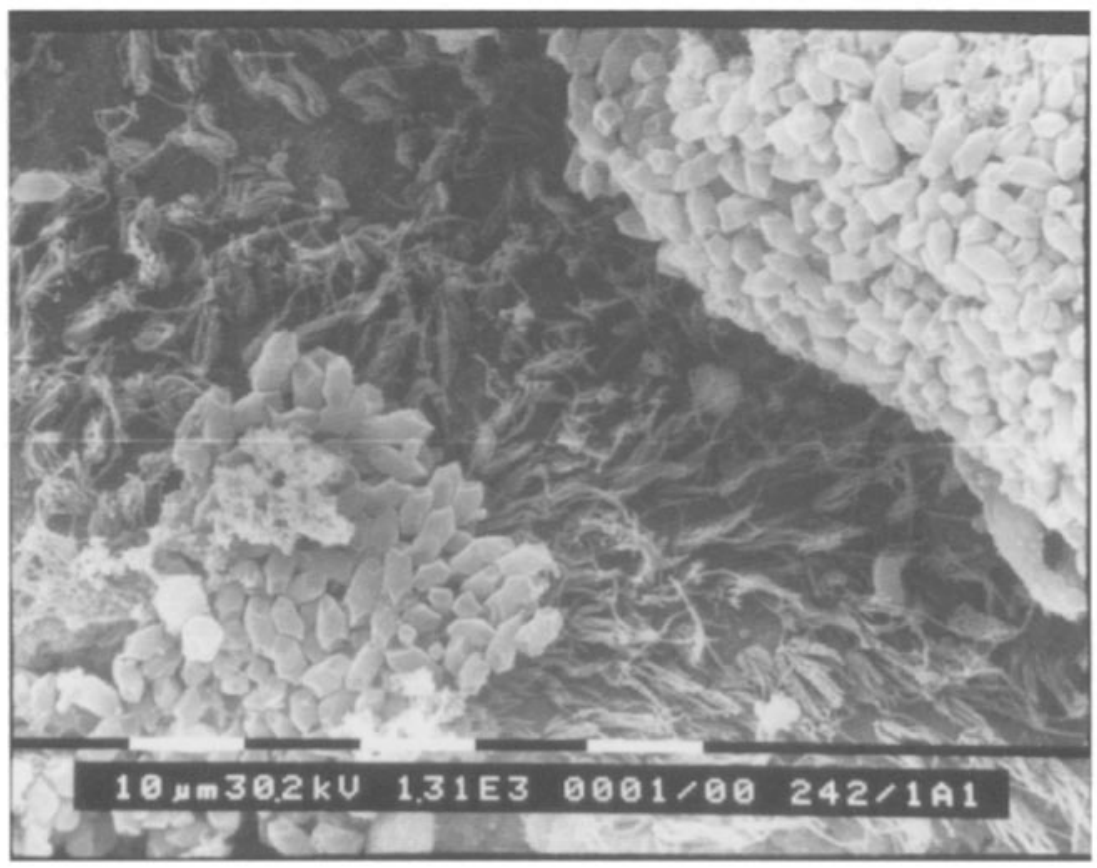

Fig. 4. Otoconia of utricular and saccular maculae in the mice are clearly detectable on the epithelial layer. 
in the vertical plane, without linear acceleration acting on the head, head tilt changes the space position of the maculae so that the gravity vector projects, on the receptor plane and bends the cilia of the sensory cells. In this case the otoconia will be dislocated in the same direction of the gravity vector component projected in the macular plane.

This mechanical system can be expressed by:

$\ddot{x}+b / m \dot{x}+k / m x=\left(1-\rho_{e} / \rho \circ\right)(g x-a x)$

where $x$ is the otoconia displacement, $g x$ is the gravity vector projection on the macula plane and $a x$ is any other linear accelerative input ( $b$ is the endolymph viscosity, $k$ is the elasic modulus of the gelatinous layer, $\rho_{e}$ is the endolymph censity, $o$ is the otoconia density and $m$ is the otoconia mass).

The total shear force, $F_{s}$ acting at the utricular otholith is: $F_{s}=g m \sin \alpha-m R^{2} \omega$. The first term represents the force created by gravity acting on the mass: (where $g$ is the gravitational acceleration); for angular tilts from prone orientation, the shear forces are: $F_{s}=g m s i n \alpha$.

During head rotation the inertial torque generated at the maculae is trivial for most frequencies of head movement. However, for higher frequencies of stimulation, this might not be true. The inertial torque is: $1=m R^{2}$.

$R$ is the radius of gyration for the effective mass $(\mathrm{m})$ of the utricular otolith. Because, in the rotatory experiments, the planes of the utricular otoliths could not be perfectly aligned with the axis of rotation, the radius of gyration could be large. The inertial torque (T) generated at the utricular otolith is: $T=$ $m R^{2} \omega$ where $\omega$ is the head angular acceleration.

During VE exposure, the angular motion perception is guaranteed by the head tracking that is the most valuable input for promoting the sense of immersion in a VR system. This artificial motion perception causes a sensory motor recalibration that can be responsible for a cue conflict between the labyrinthine and the visual system (cybersickness) and for the lack in the readaptation to the nonvirtual environment (aftereffects).

\section{Visual Afferents}

In normal subjects, vision plays a major role in the multisensory process of postural stabilisation [19]. Unlike labyrinthine stimuli, which invariably lead to the sensation of body motion, visual stimuli provide for two perceptual interpretations: self motion or object motion. Visual stabilisation of posture is crit- ically dependent on the performance of the visual system as well as on stimulus characteristics.

A decreasing of visual acuity can cause a postural unbalance and visual motion sensitivity declines from the macula towards the retinal periphery.

Moreover, there is a relationship between lateral body sways and eye-object distances as convergence and accommodation play a significant role in postural control [20]. A general improvement in postural control during the fixation of close visual targets in the grasping space is probably related to a better resolution in the detection of relative motion of head sway because of the greater retinal slip [21].

Other visual system inputs such as smooth pursuit and saccadic movements come from the visuo-vestibolo-oculomotor system and from the optokinetic reflexes [22].

In order to respect such a sophisticated system, the visual displays utilised during VE exposure would guarantee stereoscopic vision, adequate resolution, adequate brightness, and high-quality motion representations. The evaluation of the direction at which the individual's eyes are pointed out of the head is of great importance while the head position or orientation plays a minor role. This problem can be solved by eye-tracking devices (optic, electroocular, and electromagnetic) that are able to determine the direction of users' gaze and to update the visual display accordingly.

\section{Somatosensorial Afferents}

In addition to the gaze control system, there is a parallel posture control system with multiple input coming from muscular and tendinous receptors. Inputs from these receptors reach the spinal cord and the vestibular nuclei. The main role of these signals is to inform the vestibular system about the position of the different segments of our body. Moreover. the feedback control and modulation of our body movements is possible by information received from these receptors. During VE exposure, the apparent movement yielded by the visual perception does not correspond to what is registered by the proprioreceptors and a cue conflict may be present.

\section{Motor Output}

The vestibular nuclear complex processes vestibular inputs and it implements direct fast connections 
between incoming afferent information and motor output neurons.

The cerebellum is the adaptive processor that monitors vestibular performance and keeps it 'turned up'. It modulates contraction of both agonist and antagonist muscles during automatic and voluntary movements. At both locations, vestibular sensory inputs are processed in association with somatosensory and visual input [9].

The output neurons of the VOR are the motor neurons of the ocular motor nuclei, which drive the extraocular muscles. The VOR helps to maintain a stable visual image on the retina. When the head is turned in one direction, the eyes rotate in the opposite direction. This helps to maintain the visual field in the horizontal plane.

The outputs of VSR are neurons located within the anterior horns of the spinal cord gray matter, which drive skeletal muscles, but the connections are more complicated than for the VOR.

Postural control is dependent on the ability to maintain control of position and motion of the body centre mass within the base of support. Such control is a function of the organisation and integration of inputs coming from multiple sensorial sources. For example, knowledge of head orientation with respect to the gravity vector (otolith end organs) is of little use in the regulation of postural stability, unless relative trunk and limb segment positions are known. Furthermore, knowledge of how body segments are oriented one to each other is not sufficient to ensure postural stability, as an external spatial reference is still required [1]. Besides vestibular inputs, somatosensory inputs from the feet (support surface inputs) can provide an external spatial reference for body orientation and balance.

\section{Vestibular Dysfunction}

Vestibular damage can affect peripheral receptors, central pathways and motor reflexes. Regardless of the site of lesion, vestibular damage may be classified as unilateral or bilateral and, considering the onset and time course, as acute, chronic or recurrent. The behavioural changes more frequently seen in clinical practice are mainly due to damage to the peripheral vestibular organ (Labyrinth), but they are always modulated by the integration taking place in the central nervous system. This modulation is faster and easier in peripheral, unilateral, acute lesions [23].
The clinical science of vestibular deficits can be classified as static symptoms or dynamic deficits. The former are present when there is no applied vestibular stimulus other than the normal gravity force. The latter are revealed during dynamic vestibular stimuli and comprise deficits of the oculomotor response to angular and linear accelerations that occur during movements. However, the division in static and dynamic deficits is artificial because the static background activity affects the response to dynamic stimulation.

- Static symptoms

- vertigo

- spontaneous nystagmus

- ocular tilt reaction (it consists of a maintained head tilt toward the injured side, a maintained conjugate ocular torsion rolled toward the injured side, and skew deviation toward the injured side)

- Dynamic deficits have been recognised only since measures of dynamic vestibular function (Norre. 1994), have been introduced.

- oscillopsia (unstable vision during waking)

- head impulse rest (poor vestibular response during head impulse rotation)

- caloric response (poor vestibular response during caloric stimulation)

- rotational response (poor vestibular response during roto-acceleratory stimulation)

\section{Vestibular Assessment}

Diagnosis of vestibular damage should be able to identify if labyrinthic or central pathways are involved. If the labyrinth is involved, the clinician has to identify the affected side (right or left), the entity of the lesion, and to define the time course of the deficit. This last point is important, particularly in peripheral unilatera! lesions where vestibular compensation takes place in order to reduce static and dynamic symptoms [24]. Vestibular compensation always occurs in vestibular diseases, and is usually complete only in unilateral stabilised lesions. In these cases, patients do not complain of vertigo, therefore the level of vestibular compensation influences vestibular diagnosis. The identification of the affected subsystem in vestibular damage is not always easy due to the plasticity of the central nervous system, especially when many subsystems are involved (as in elderly and old 
patients). Nowadays, the instrumental diagnostic tools are based on the study of reflexes elicited by non-specific and non-physiologic stimuli administered to the individual's peripheral receptor. Furthermore, as the obtained responses are centrally integrated, their sensibility and specificity are reduced.

Vestibular function has been evaluated for many years by studying posture and ocular movements on a merely clinical basis. In the last decades, more accurate instrumental tools have been introduced and now allow for a more precise quantification of VOR and VSR outputs, reflecting vestibular system function that is crucial in vestibular rehabilitation.

Video and electro-oculography measure eye movements, both spontaneous and induced, by positional manoeuvres or in response to positional, rotational and caloric stimulation. While video-oculography records and visualises ocular movements by means of an infrared camera, electro-oculography evaluates changes in electrical potential by placing electrodes on the skin around the eye record. An electrical dipole exists along the long axis of the eye, with the retina retaining a negative potential. Electrodes above, below and lateral to the eye record change in potential induced by eye movements. Pathological findings can suggest a labyrinthine or central vestibular pathways dysfunction but they seldom localise the lesion or identify the pathogenesis.

Posturography evaluates the postural control by recording the body sway in various emironmental conditions. These conditions allow a quantitative analysis of the role of the different sensorial afferences in the postural control since the subject may be deprived of information coming from the visual and proprioceptive systems by moving the visual surround and/or the platform proportionally to the body sway. A sophisticated apparatus used to test vestibular-mediated postural function is the Equi-Test Dynamic Posturography System by NeuroCom, which consists of a dual force plate to control foot support and a controllable visual surround reference. Force transducers measure vertical and horizontal output of the subject's feet. The subject's centre of gravity is monitored and used to estimate the body sway during testing. Dynamic Posturography is able specifically to reduce the available sensorial afferences (visual, labyrinthine, proprioceptive), and identify the pathological sensorial system by calculating the score in these different environmental conditions. Moreover, when a sensorial deficit is present. Dynamic Posturography is able to evaluate the new hierarchy of the sensorial cues. On the whole, it can be stated that this method mainly relies on the postural effects following an artificial deprivation of sensorial inputs $[25,26,27]$.

\section{Vestibular Rehabilitation}

The efficiency of the vestibular system relies mainly on the integration of various sensorial inputs. These inputs are redundant so that any individual may use them in a different hierarchy relative to previous experiences, temporary needs and to age. This physiologic plasticity is enhanced if a vestibular lesion occurs. In Fact, the lack of one or more sensorial afferences induces a rearrangement of the vestibular system strategy for maintaining postural control and stable vision.

A rearrangement of the hierarchy of the vestibular afferences may be induced in normal subjects in order to reduce adverse effects that mighc occur during a specific sensorial overstimulation. This is the case of ice skaters, where a decrease of the labyrinthine gain is a result of training, and of Air Force pilots where a visual-vestibular conflict can induce motion sickness [28].

The training in flight simulators can artificially induce the rearrangement of the hierarchy of the vestibular system by enhancing the saliency of visual cues $[29,30,31,18,22]$. After unilateral vestibular loss, the relatively rapid and apparently complete behavioural recovery is justified by vestibular compensation. It is not a single process that recovers completely at a rapid rate but the result of several subprocesses taking place at different levels, at different rates and in two main ways: adaptation and/or substitution.

Adaptation is represented by a reduced peripheral gain during the acute phase at the contralateral vestibular nuclear level without external stimulation. This consists of decreased firing at the contralateral vestibular nuclei level immediately after a unilateral labyrinthine acute lesion, while in the experimental animal after a few hours neural firing slowly recovers in the ipsilateral nuclei. Mechanisms underlying these adaptation phenomena are still largely unknown.

Substitution is necessary in the presence of a sensorial deficit and occurs on the redundancy of the semsorial afferences to the vestibular system (visual, labyrinthine, somatosensorial) [32]. It is 
known that this integration takes place at the vestibular nuclear complex and cerebellar levels. These structures receive information from the different sensorial systems and may influence the motor outputs. When specific sensorial damage is present, the other cues can be enhanced, thus yielding an almost normal output. Therefore, the functional recovery following a peripheral vestibular lesion or disorder is dependent on these adaptive central nervous system processes that require adequate visual and somatosensory feedbacks [33]. From a clinical standpoint, however, it is common for patients with peripheral vestibular lesions to experience persistent signs and symptoms that may include postural imbalance, gaze instability during head movements and vertigo. In patients with chronic vestibular dysfunction, compensation for the loss or disruption of peripheral vestibular inputs can be effectively induced by exercises that provide sensory feedback appropriate for behavioural changes involving sensory substitution or sensory motor reorganisation [34]. In this regard, after an acute peripheral vestibular lesion, VOR compensation and recovery of postural reftexes were delayed and substantially reduced when experimental animals were deprived of vision and kept in small cages or castled to restrict or prevent head and limb movements. On the other hand, it has been demonstrated in unilateral labyrinthectomised cats that recovery of VOR gain symmetry is facilitated by daily exercises. Functional recovery of gaze stabilisation during combined eye-head movements is in fact achieved by different sets of strategies and mechanisms. These include enhancement of the cervico-ocular reflex, modification of the velocity and amplitude of the saccadic eye movements to targets, and a decrease in the velocity of the head movement. Oculomotor control is severely affected after bilateral vestibular damage that causes the absence of VOR. Such a deficit is usually rearranged by several substitution mechanisms concerning visual information (retinal slip, optokinetic reflex (OKR)), cervical proprioception (cervico-ocular reflex (COR). cervico-collic reflex (CCR)) and other somatosensory inputs [35]. A recalibration of left-right difference in VOR gain could contribute to the recovery of gaze stabilisation. The manoeuvres and positional changes that provoked symptoms of vertigo and dizziness are commonly employed for vestibular rehabilitation $[36,37,38]$. The value of these vestibular exercises is based on the concept that repeated exposure to provocative stimuli will result in an erroneous signal for the CNS that provides for a correction by means of a sensory reorganisation. The results showed that the patients who regularly performed the exercises experienced a reduction in intensity and duration of vertigo, and a progressive reduction in the number of manoeuvres that provoked vertigo. Investigations about postural control have demonstrated the importance of visual [39], somatosensory and vestibular inputs to the normal regulation of postural stability [40]. Support for the use of exercise to promote recovery of postural stability following peripheral vestibular lesions comes from a number of studies $[17,41,42]$. These evaluated the effects of exercise on balance performance in monkeys following unilateral and bilateral labyrinthectomies. A significant enhancement in locomotion balance performance in the exercise group was observed as compared to a nonexercise control group. The implication of the above-mentioned studies seems to be that it is possible to facilitate and achieve optimal functional recovery following peripheral vestibular damage or dysfunction. Therefore, goaldirected activities under combinations of various visual and somatosensory conditions should be encouraged, even though the activities may temporarily aggravate accompanying symptoms of vertigo and dizziness.

\section{Personal Research}

Postural controf in normal subjects is due mainly to visual inputs [39]. Virtual environments (VEs) can induce a conflict between motion perception produced by the visual system with vestibular and proprioceptive inputs indicating a static condition. This 'neural store' vestibular-visuai-proprioceptive conflict is reduced or even absent if an acute sensorial deficit is present while postural control is affected.

Postural studies during specific VE exposure could allow the evaluation of the postural strategy and the role of each cue (visual, labyrinthine, and proprioceptive). In VE, the external spatial reference for body orientation and balance can be artificially modulated in order to promote the reorganisation of the multiple sensory input reducing the erroneous signals and enhancing the avallable informations.

The purpose of this paper was to evaluate the reliability of VE exposure in the assessment of vestibular disorders and its possible role on vestibular rehabilitation. 


\section{Materials and Methods}

The rearrangement of the hierarchy of the postural cues was evaluated in 105 subjects when a sensorial deprivation was artificially induced.

Patients were divided into five groups according to pathology, and compared to 50 normal subjects:

1. Sudden loss of unilateral vestibular function $(n=15)$;

2. Compensated loss of unilateral vestibular function $(n=15)$;

3. Bilateral chronic loss of vestibular function $(n=15)$;

4. Patients affected by Congenital Nystagmus (CN) $(n=15)$;

5. Patients affected by peripheral sensorial polineuropachy $(n=45)$.

This study was performed with the immersion of the patients in an artificial environment where the information coming from the visual, the labyrinthine and somatosensorial systems was artificially altered in order to evaluate their specific importance on postural control. The subject was standing on a dual forceplate enclosed by a visual surrounding with the head in the upright position. The test was performed in a darkened room with the visual surroundings lit by a fixed fluorescent light in order to ensure a constant level of illumination. The dual forceplate records the vertical forces between feet and ground, as well as the horizontal shear forces, thereby allowing estimation of the position of the swaying body. Six different test conditions were performed, each lasting 20 seconds and repeated three times in order to obtain more reliable values:

1. Eyes open. Platform, surface and visual surrounding steady.

2. No data concerning specific deficits can be detected, as in this condition all the sensorial afferences of the vestibular system are available.

3. As in condition 1, but subject's eyes are closed. A marked decrease in score compared to condition 1 suggests a postural strategy based on visual cues, due to the labyrinthine and/or somatosensorial deficit, although patients with compensated vestibular deficit might score aimost normally.
4. Platform still steady, visual surrounding rotates on an axis colinear with the subject's ankle joint in direct proportion to the anteroposterior sway of the subject's centre of gravity. Under such conditions the subject cannot perceive changes of the visual field, while somatosensorial and labyrinthine receptors perceive the body sways. Therefore no visual information can be obtained and a sensory conflict is present.

5. Visual surrounding steady, eyes open and platform rotates proportionally to the patient's sway so that changes of the subject orientation with respect to the platform are abolished. In this condition no somatosensorial informations are available and therefore visual and labyrinthine cues are enhanced.

6. Visual surround steady, eyes closed and platform rotates as in condition 4. In this condition the role of labyrinthine cues is enhanced.

7. Eyes open, platform and visual surround rotate proportionally to the antero-posterior body sway. In this condition the role of vestibular cues is enhanced since visual and somatosensorial afferences are inaccurate and do not yield information about the postural swaying

The Equilibrium Scores (ES) and Strategy Score (SS) were worked out for each condition (see Table 2 and Table 3 respectively).

ES indicates the range of sway angle with respect to the vertical and is computed for each condition. It quantifies how well the patient's sway remains within the expected angular limits of stability during each SOT condition. The following formula is used to calculate the equilibrium score:

EQULIBRIUM: $\left[12.5^{\circ}-(\theta \max -\theta \min ) / 12.5^{\circ}\right] \times 100$

SS quantifies the ankle and hip movements the patient uses to maintain equilibrium during each 20-second trial accordingly to formula:

\section{MOVEMENT STRATEGY: \\ $[1-(\mathrm{SH}$ max-SHmin/25)] $\times 100$}

In this formula, $25 \mathrm{lbs}$ is the difference measured between the greatest shear force (SHmax) and the lowest shear force (SHmin) generated by a test group of normal subjects. A score near 100 indicates that ankle strategy is predominant in maintaining equilibrium, while a score near 0 shows that hip strategy is predominant. 
Table 2. Equilibrium scores in all groups in the six different test conditions.

\begin{tabular}{lllllll}
\hline $\begin{array}{l}\text { Test condition } \\
\text { normal }\end{array}$ & Normal & Acute $\mathrm{m}$ & Chronic $\mathrm{m}$ & Chronic b & Congenital Ny & Neuropathy \\
\hline 1 & $92.8 \pm 1.2$ & $87.8 \pm 2.9$ & $90.4 \pm 1.4$ & $91.8 \pm 2.2$ & $92.3 \pm 1.2$ & $93.7 \pm 1.1$ \\
2 & $92.5 \pm 1.5$ & $83.5 \pm 5.9$ & $91.7 \pm 1.6$ & $89.5 \pm 3.1$ & $93 \pm 1.3$ & $87 \pm 6.2$ \\
3 & $91.3 \pm 2.2$ & $85.3 \pm 6.1$ & $90.4 \pm 2.3$ & $87.3 \pm 4.7$ & $92.5 \pm 2.1$ & $88.6 \pm 4.2$ \\
4 & $82.5 \pm 5.9$ & $82.3 \pm 21.1$ & $80.7 \pm 5.7$ & $82.5 \pm 5.3$ & $71 \pm 7.2$ & $80.4 \pm 6.1$ \\
5 & $67.7 \pm 7.0$ & $43.6 \pm 22.2$ & $63.7 \pm 7.2$ & $53.6 \pm 16.2$ & $59 \pm 15.6$ & $61.5 \pm 8.7$ \\
6 & $65.3 \pm 7.9$ & $39.3 \pm 22.8$ & $60.8 \pm 2.1$ & $45.8 \pm 18.1$ & $58.3 \pm 10.1$ & $58.5 \pm 9.9$ \\
\hline
\end{tabular}

Table 3. Sensory andysis for all groups.

\begin{tabular}{lllllll}
\hline $\begin{array}{l}\text { Test condition } \\
\text { normal }\end{array}$ & Normal & Acute $m$ & Chronic $m$ & Chronic b & Congenital Ny Neuropathy \\
\hline Composite & $79.2 \pm 3.5$ & $63 \pm 12.8$ & $76 \pm 4.1$ & $62 \pm 9.2$ & $73 \pm 4.2$ & $69 \pm 9.4$ \\
Somatose & $98 \pm 1.1$ & $85 \pm 21.4$ & $95 \pm 1.8$ & $89.5 \pm 13.1$ & $93 \pm 1.8$ & $53 \pm 17.1$ \\
Visual & $89 \pm 6.1$ & $85.3 \pm 20.2$ & $90.4 \pm 5.4$ & $78 \pm 18.4$ & $49 \pm 23.1$ & $88.6 \pm 5.7$ \\
Vestibular & $72 \pm 6.9$ & $47 \pm 24.1$ & $71 \pm 6.3$ & $51 \pm 21.1$ & $74 \pm 5.4$ & $80.4 \pm 6.2$ \\
\hline
\end{tabular}

The Composite Score is a weighted computation of the sensory organisation test scores and yields an overall evaluation of the scores obtained in the six conditions.

The Sensory Analysis (SA) shows the relative contribution of the different sensory afferents and is calculated by the equilibrium score obtained in the different test conditions.

Statistical analysis: all results are expressed as means and compared to normal groups. Differences were considered significant if $p<0.05$.

\section{Results of the Sensory Organisation Test}

Normal scores were recorded in all subjects of the control group. Concerning patients, the scores obtained in the five groups showed the following features (tables 2.3 and figs 5,6):

Labyrinthine Pathology: Different results were observed according to the uni- or bilaterality of the lesion and to the onser modality. In patients affected

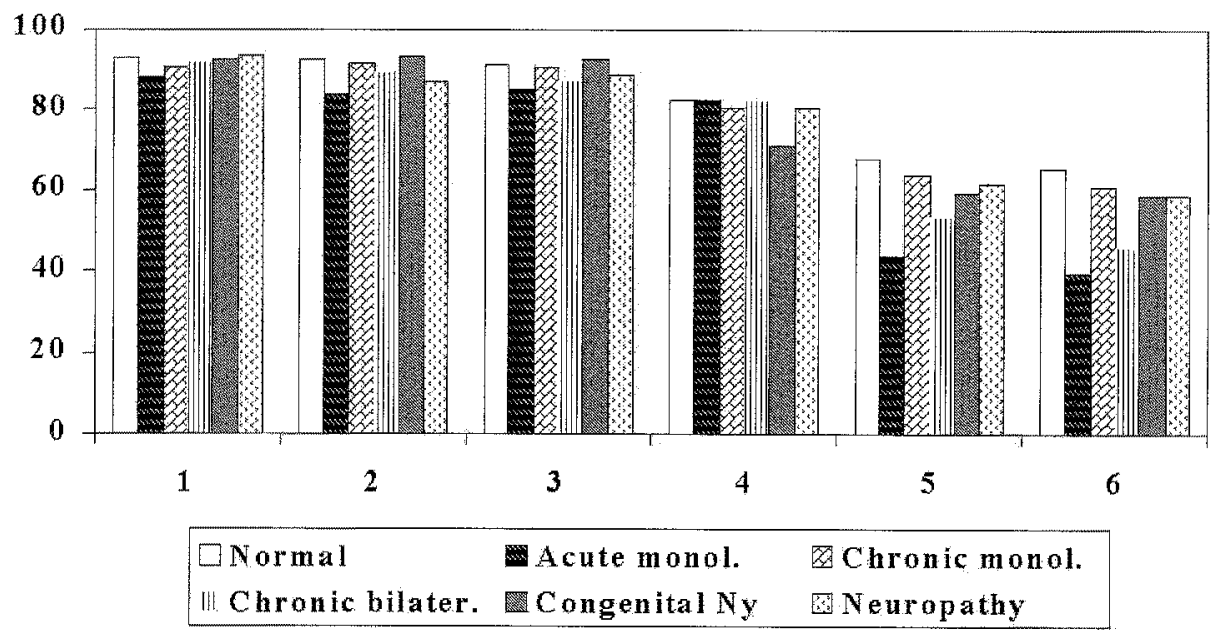

Fig. 5. Sensory equilibrium scores in the six different test conditions 


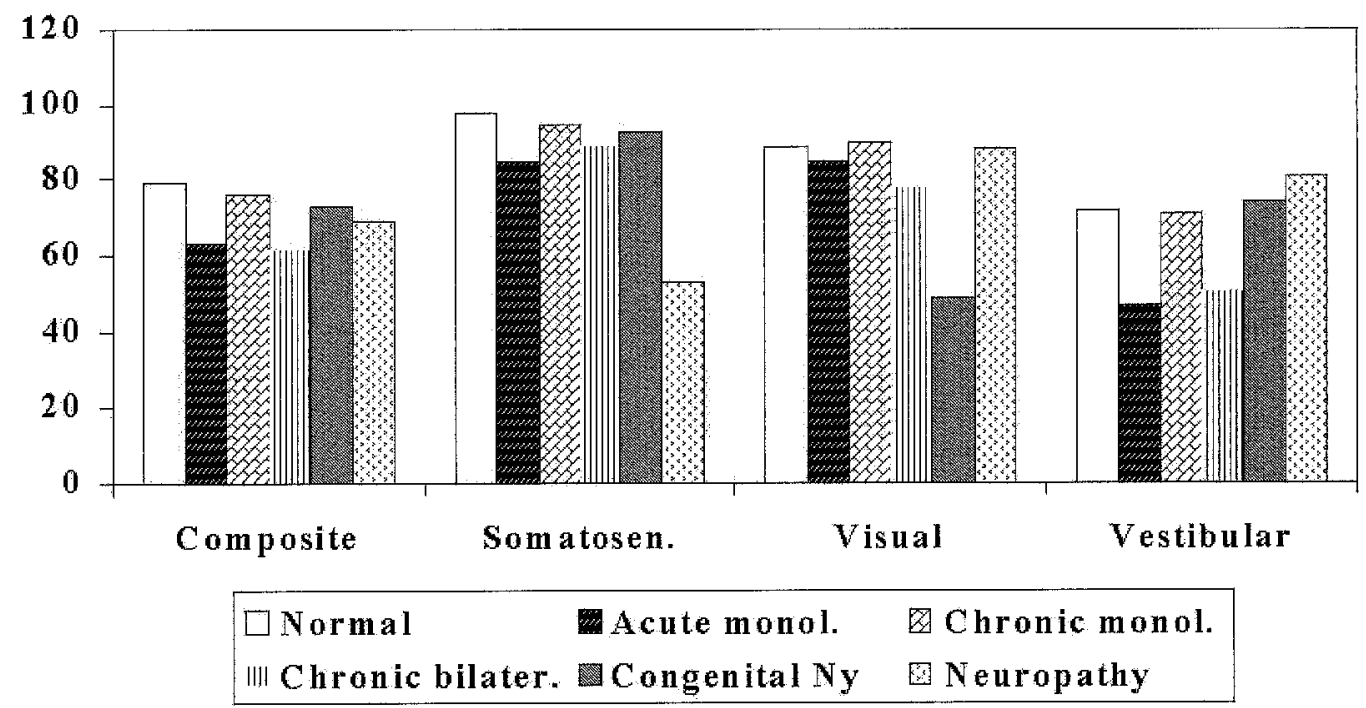

Fig. 6. Sensory analysis scores in the different groups.

by sudden unilateral loss of vestibular function, a clear-cut score reduction was observed in test conditions 5 and 6 , the most dependent on labyrinthic inputs. Sensory analysis obviously confirmed the labyrinthic nature of the deficit. Moreover, the overall composite score was markedly reduced in comparison to the control group. No deficits were recorded if the unilateral labyrinthic loss was compensated, with normal scores in all the six conditions, due to vestibular compensation. On the contrary, in patients with chronic bilateral vestibular dysfunction, once again lower scores were recorded in comparison to control in test conditions 5 and 6 . The artificial reducing of the somatosensory and visual cues in sudden unilateral and chronic bilateral groups determined a greater effect on balance than it had on compensated monolateral labyrinthic deficit or in normal subjects.

Patients affected by Congenita Nystagmus: Data obtained in this group are particularly intriguing, due to the fact that patients affected by $\mathrm{CN}$ are not able to use visual cues for postural control. In fact, the overall postural control was basically impaired when visual cues were present (conditions 1 and 4) and this impairment was even more enhanced in $\mathrm{CN}$ than in the normal control group when somatosensorial cues were experimentally reduced (conditions 4, 5 and 6). On the contrary, removal of visual cues (condition 2) did not induce a deterioration of postural control as seen in normal subjects. Our data clearly outline that in $\mathrm{CN}$ an impairment of the postural balance is present especially when the postural control relies mainly on visual cues. Moreover, a decrease in accuracy of the somatosensory cues has a proportionally greater effect on balance than it has on normal subjects.

Diabetic peripheral neuropathy: A deficit in postural control was clearly recognisable in the strategy score where a shift from physiological ankle control to hip postural control was present due to the peripheral polineuropathy. These data showed a linear correlation with the neurophysiological investigations such as nerve conduction. The sensory analysis showed a specific somatosensorial deficit as shown by the lower scores during inaccurate visual afferences.

\section{Conclusions}

Postural control is achieved by the interaction between various sensorial subsystems (labyrinthine, visual and somatosensorial). The recorded spatial information is often redundant but this redundancy is useful in order to allow the vestibular system to choose the most significant in any circumstance, static or dynamic. Therefore, this redundancy allows the subject to maintain a satisfying postural control in the presence of postural deficit affecting a vestibular subsystem after the readaptation processes have taken place.

Our data confirm the role exerted by the other subsystem cues when one of them is lacking. In all patients we found an increment of the available sensorial afferences with a subsequent hierarchical reorganisation. 
Congenital nystagmus represents a pure mode of reduction of visual cues since birth. Movements of visual field lead the central nervous system to reduce the role of visual cues in the hierarchy of postural control [43]. This decrease on the visual cues induced in our patients an increment of the proprioceptive and labyrinthine information, as shown by results obtained in the test conditions with visual input not available or artificially reduced (2.5 and 6).

Normal subjects and patients affected by labyrinthine damage or diabetic neuropathy behave differently.

In diabetic neuropathy, information from foot, muscular and tendineous proprioceptors are altered by the neural structural damage and therefore no information coming from these receptors is avallable. In this group, visual and labyrinthine systems play a major role, as shown by results obtained in conditions with altered proprioceptive cues (4. 5 and 6).

Much more puzzling are results obtained in labyrinthine pathology, according to uni- or bilaterality and time course of the lesion. An acute unilateral damage is characterised by a first phase of vestibular decompensation followed by a resetting of the vestibular system. The functional deficit present in the first phase induces the impossibility of maintaining postural control, especially if visual and proprioceptive cues are reduced (conditions 5 and 6). In the second phase, clinical and instrumental signs of vestibular unbalance progressively disappear, due to incoming compensation. If vestibular damage is chronic and bilateral, labyrinthine cues are almost lacking and the other two subsystems increase their role (substitution). Substitution mechanisms are not able to take the place of labyrinthine cues completely and the reset system is not efficient enough in challenging situations, such as walking in darkness, where substitutory inputs are reduced.

Our model evaluates the three subsystems independently and is able to identify the one dysfunction. Moreover, it evaluates the substituting role of the other subsystems. This plasticity can be exploited during an immersion in a virtual environment to stimulate and accelerate adaptation by artificially modulating sensorial information. Such a mechanism is confirmed in our patients where a pure single sensorial deficit is compensated in order to obtain an acceptable postural control.

The exposition of a patient to VE may take up a role in both diagnosis and rehabilitation [44]. Concerning rehabilitation. VE exposure is able to speed up vestibular compensation by changing vestibulo-oculomotor (VOR) and vestibulo-spinal (VSR) reflexes.

Rizzo and Buckwalter [14] stated that 'VR offers the potential to develop human testing and training environments that allow for the precise control of complex stimulus presentations in which human cognitive and functional performance can be accurately assessed and rehabilitated.' Some authors have used VE exposure in the partial rehabilitation of patients affected by severe brain injury [45]. VE exposure outcomes seem much more clinically salient when dealing with the vestibular system because a continuous rearrangement of different information is nearly always present and is physiologically due to environmental and age-related changes. Therefore, it seems reasonable to employ $V R$ in order to accelerate the compensation of an acute loss of labyrinthine function where adaptive modifications of the vestibulo-ocular and vestibulospinal reflexes are more important. In this case, it appears that VE exposure should be targeted specifically at overstimulating the residual labyrinthine function, rather than the visual one.

VE exposure side effects are very similar to symptoms seen in vestibular damage, due to the incongruence among the different perceptive modalities, namely visual, proprioceptive and labyrinthine. that regulate postural control.

On the whole, VE induces a sensorial conflict by changing relationships between a redundant visual input and labyrinthine and proprioceptive responses. As a consequence, postural strategy has to change in order to adapt. itself to a new 'reality'. Similar events take place in the presence of a deficit of a vestibular subsystem and the whole system should be reseted.

Therefore, VE exposure could enhance the physiological compensatory mechanisms of substicution in chronic bilateral vestibular deficit. In fact, $V E$ can modulate the external spatial reference for body orientation and balance, and can promote the reorganisation of the multiple sensory input, thereby enhancing the available sensorial information. The vestibular system appears, then, as a potential and very interesting field for VR applications in the areas of vestibular assesment and rehabilitation.

Our data show that VE exposure may help to detect the deficient sensorial subsystem as it allows an artificial and selective reduction or enhancement of each sensorial cue involved in postural control.

Concerning vestibular rehabilitation it has been shown that various visual, sensory and motor manipulations can affect the rate of recovery. VE 
exposure seems then potentially even more useful in vestibular rehabilitation, since the experiences gained during VE exposure are directly transferable to the real world.

\section{References}

1. Cobb SV. Measurement of postural stability before and after immersion in a virtual environment. Appl. Ergon. Feb 1999: 30(1): 47-57

2. Kolasinski EM, Gilson RD. Ataxia following exposure to a virtual environment. Aviat Space Environ. Med. March 1999; 70(3 PL 1): 264-269

3. Stanney KM. Hash P. Locus of user-initiated control in virtual environment: influences on cybersickness. Presence: Teleoperators and virtual environments, 1997

4. Reason JT. Motion sickness: a special case of sensory rearrangement. Advancement in Science 1970; 26; 386-393

5. Regan E. Price KR. The frequency of occurrence and severity of side effects of immersion virtual reality. Aviat. Space Environ. Med. 1994; 65: 527-530

6. Lackner JR, Graybiel A. Visual and postural motion aftereffects following parabolic flight. Aviat. Space Environ. Med. March 1980; 51(3): 230-233

7. Howarth PA, Finch $M$. The nauseogenicity of two methods of navigation within a virtual environment. Appl. Ergon. February 1999; 30(1): 39-45

8. Kennedy RS, Stanney JM, Ordy JM. Dunlap WP. Virtual reality effects produced by head-mounted display (HMD) on human eye-hand coordination, postural equilibrium, and symptoms of cybersickness. Society for Neuroscience. Abstracts 1997; 23: 772

9. Van Beers RJ, Sittig AC, Denier van der Gon JJ. How humans combine simultaneous proprioceptive and visual position information. Exp. Brain Res., September 1996; 111(2): 253-261

10. Hettinger $U$. Visually induced motion sickness in virtual environments. Presence: teleoperators and virtual environments 1992; 1: 306-307

11. Precht $W$. Recovery of some vestibulocular and vestibulospinal functions following unilateral labyrinthectomy. Prog. Brain Res. 1986; 64: 381

12. Di Zio P. Lackner JR. Spatial orientation, adaptation, and motion sickness in real and vitual environments. Presence: teleoperators and virtual environments $1992 ; 1: 323$

13. Kennedy RS. Stanney KM. Postural instability induced by virtual reality exposure: development of certification protocol. International Journal of Human Computer Interaction 1996; 8(1): 2547

14. Rizzo AA. Buckwalter JG. Virtual reality and cog nitive assessment and rehabilitation. The state of the art. In G Riva, ed: Psycho-neuro-physiology assessment and rehabilitation in virtual environments: cognitive, clinical, and human factor in advanced human computer interaction. Amsterdam: IOS Press. 1997; 123-145

15. Norré ME. Habituation, sensory substitution and structural reorganization in vestibular compensation. In: Equilibrium disorders: brainstern and cerebellar pathology. Cesarani A, Alpini D, eds. Berlin: Springler verlag, 1994; 199-204

16. Norrè ME. Relevance of function test in the diagnosis of vestibular disorders. Clin. Otolaryngol. 1994; 19: $433-440$

17. Igarashi. Physical exercises and acceleration of vestibular compensation. In: Vestibular compensation. Lacour M, Toupet M. Denise P, Cristen $Y$, eds. Amsterdam: Elsevier, 1989; 101-105

18. Potvin AR, Sadoff M, Bilfingham J. Motion sickness and otholith sensitivity: a pllot study of habituation to linear acceleration. Aviat. Space Environ. Med. November 1977; 48(11): 1068-1075

19. Paulus W. Hawken M, Ouintern J. Straube A, Krafczyk S. Botzel K. Brandt T. Multisensory versus monosensory stabilization of posture. Agressologie 1988; 29(10): $699-703$

20. Edwards AS. Body sway and vision. J. Exp. Psychol. $1946 ; 36: 526-535$

21. Brandt $T$. The relationship between retinal image slip. oscillopsia and postural imbalance. in: Functional basis of ocular mocility disorders. Lennerstrand $G$; Zee DS. Keller EL. eds. Oxford, New York: Pergamon Press $1982 ; 379-385$

22. Hu SQ, Stern RM, Koch KL. Effects of pre-exposure to a rotating optokinetic drum on adaptation to motion sickness. Aviat. Space Environ. Med. January $1991 ; 62(1): 53-6$

23. Cooksey TE. Vestibular injuries. Proc. R. Soc. Med $1945,39: 270-273$

24. Curthoys IS, Halmagy MG. Vestibular compensation a review of the oculomotor, neural, and clinical consequences of unilateral vestibular loss. Journal of Vestibular Research. 1995; 5(2): 67-107

25. Straube A, Paulus W, Ouintern J, Brandt T. Visual ataxia induced by eye movements: posturographic measurements in normals and patients with ocular motor disorders. Clin. Vision Sci. 1989: 4: 107-113

26. Voorhes RL. The role of dynamic posturography in neurotologic diagnosis. Laryngoscope 1989; 99: $995-$ 1001

27. Paulus W, Straube A, Brandt T. Visual postural performance after loss of somatosensory and vestibular function. J. Neurol. Neurosurg. Psychiat. 1987; 50: 1542-1545

28. Graybiel A. Lackner JR. Motion sickness: acquisition and retention of adaptation effects compared in three motion environments. Aviat. Space Environ. Med. Apri 1983: 54(4): 307-311

29. Peterka RJ, Benolken MS. Role of somatosensory and vestibular cues in attenuating visually induced human postural sway. Exp. Brain. Res. 1995; 105: 101-110

30. Kennedy RS, Fowkes JE. Lilienthal MG. Postural and performance changes following exposure to flight simulators. Aviat. Space Environ.Med. October 1993; 64(10): 912-920

31. Fowkes JE, Kennedy RS, Hertinger U, Harm DL Aviat Space Environ. Med. July 1993; 64(7): 612-618

32. Szturm T, Ireland DJ, Lessing-Tuner M. Com parison of different exercise programs in the rehabilitation of patients with chronic perpheral vestibular disfunction. Journal of Vestibular Research 1994: 4(6): $461-479$

33. Vitte $E$, Semont A, Berthoz A. Repeated optokineric stimulation in conditions of active standing facilitates 
recovery from vestibular deficits. Exp. Brain Res. 1994: 102(1): 141-148

34. Bles W, Jong JMBV, de Wit G. Somatosensory compensation for loss of labyrnthine function. Acta Otolaryngol. (Stockh). 1984: 97: 213-221

35. Grant SC, Magee LE. Contribution of proprioception to navigation in virtual environments. Hum. Factors September 1998; 403(3): 489-497

36. Telian SA, Shepard NT. Update on vestibular rehabilitation therapy. Otolaryngol Clin. North Am. 1996; 29: $359-371$

37. Shumway-Cook A, Horak FB. Rehabilitation strategies for patients with vestibular deficits. Neurol Clin. 1990; 8: $441-457$

38. Horak FB, Jones-Rycewicz C, Black FO, ShumwayCook A. Effects of vestibular rehabilitation on dizziness and imbalances. Otolaryngol Head and Neck Surg. 1992: 106: 175-180

39. Paulus W. Straube A, Brandt T. Visual stabilization of posture: physiological stimulus characteristics and clinical aspects. Brain 1984; 107: 1143-1163

40. Shepard NT, Telian SA. Smith-Wheelock M. Raj A. Vestibular and balance rehabilitation therapy. 1993 102: $198-204$
41. Herdman SJ. Role of vestibular adaptation in vestibular rehabilitation. Otolaryngol. Head and Neck Surg. 1998; 119: 49-54

42. Asai M. Effects of vestibular rehabilitation on postural control. Acta Otolaryngol. (Stockh). 1997 supp; 528 : $116-120$

43. Dieterich M, Brandt T. Impaired motion perception in congenital nystagmus and acquired ocular motor palsy. Clin. Vision Sci. 1987; 1: 337-345

44. Kuhlen T, Kraiss KF, Dohle C. Hefter H. Freund HJ Virtual holography in diagnosis and therapy of sensorimotor disturbances. In: Health care in information age; Sieburg H. Weghorst $S$ and Morgan K. Eds Amsterdam los Press 1996; $184-193$.

45. Riva G. Virtual realicy in neurosciences: a survey. In Virtual environment in clinical psychology and neurosciences. Riva G. Wiederhold BK, Molinari E, eds. Amsterdam: IOS Press, 1998

Correspondence and offprint requests: Stefano Di Girolamo. Clinica Otorinolaringoiatrica. Universito Cattolica del Sacro Cuore, Largo A. Gemelli. 00168 Rome, italy.Email: sdigirolamo@m.unicatt.it 